González-Espinosa, S.; García-Rubio, J.; Feu, S.; Ibáñez, S.J. (2020) External Load in Basketball According To Game Situation and Methodology. Revista Internacional de Medicina y Ciencias de la Actividad Física y el Deporte vol. 20 (79) pp. 395-417 Http://cdeporte.rediris.es/revista/revista79/artcarga1168.htm

DOI: http://doi.org/10.15366/rimcafd2020.79.002

\title{
ORIGINAL
}

\section{CARGA EXTERNA SEGUN LA SITUACIÓN DE JUEGO Y METODOLOGÍA EN BALONCESTO ESCOLAR}

\section{EXTERNAL LOAD IN BASKETBALL ACCORDING TO GAME SITUATION AND METHODOLOGY}

\author{
González-Espinosa, S. ; García-Rubio, J. ${ }^{2}$; Feu, S. ${ }^{2}$ e Ibáñez, S.J. ${ }^{3}$ \\ ${ }^{1}$ Estudiante de doctorado, Facultad de Ciencias del Deporte de la Universidad de Extremadura \\ (España) sgones@unex.es \\ ${ }^{2}$ Doctor por la Universidad de Extremadura. Profesor de la Facultad de Ciencias del Deporte de \\ la Universidad de Extremadura (España) jagaru@unex.es, sfeu@unex.es \\ ${ }^{3}$ Catedrático por la Universidad de Extremadura. Profesor de la Facultad de Ciencias del Deporte \\ de la Universidad de Extremadura (España) sibanez@unex.es
}

\section{AGRADECIMIENTOS O FINANCIACIÓN}

Este trabajo ha sido parcialmente subvencionado por la Ayuda a los Grupos de Investigación (GR18170) de la Junta de Extremadura (Consejería de Economía e Infraestructuras); con la aportación de la Unión Europea a través de los Fondos Europeos de Desarrollo Regional (FEDER). Agradecer al centro educativo y los estudiantes participantes por colaborar en este trabajo.

Código UNESCO / UNESCO code: 5899 Otras especialidades pedagógicas, Educación Física y Deporte / Physical Education and Sport.

Clasificación Consejo de Europa / Council of Europe classification: 4. Educación Física y deporte comparado.

Recibido 13 de agosto de 2018 Received August 13, 2018

Aceptado 17 de noviembre de 2018 Accepted November 17, 2018

\section{RESUMEN}

El objetivo fue caracterizar y comparar la carga externa en sesiones de educación física según la situación de juego en dos metodologías de enseñanzaaprendizaje diferentes. Se emplearon dos programas de intervención diseñados y validados, Direct Instruction y Tactical Game Approach. La muestra estuvo compuesta por 40 tareas. Participaron 70 alumnos de entre 10 y 12 años de un centro de educación primaria de España. Las variables analizadas fueron la situación de juego y la carga externa. Los resultados muestran que los estudiantes de la metodología Tactical Game Approach obtienen valores más 
altos de carga externa que los de la metodología Direct Instruction. Además, la carga externa disminuye cuando aumenta la complejidad de la situación de juego. La intensidad de las tareas del método Tactical Game Approach permite alcanzar los objetivos de actividad física recomendados por la Organización Mundial de la Salud y que garantizan la salud de los estudiantes.

PALABRAS CLAVE: Educación Física, Tactical Game Approach, Baloncesto, Contexto escolar

\section{ABSTRACT}

The aim was to characterize and compare the external training load according to the game situation in physical education in two different teaching-learning methodologies. Two unit was design and validated with a Direct Instruction methodology, and the other with a Tactical Game Approach methodology. The study sample consisted of 40 tasks. The study involved 70 students between 10 and 12 years of age in a primary school in Spain. The analyzed variables were the game situation and the external load. The results show that students of the Tactical Game Approach methodology obtain higher values of external training load than students of the Direct Instruction methodology. In addition, the external training load decreases when the complexity of the game situation increases. The intensity of the tasks of the Tactical Game Approach method allows reaching the physical activity objectives recommended by the World Health Organization and guarantee the health of the students.

KEYWORDS: Physical education, Tactical Game Approach, Basketball, School Context.

\section{INTRODUCCIÓN}

La educación tiene como objetivo el desarrollo integral de los alumnos. Este desarrollo integral tiene varios componentes que hacen al alumno desarrollarse en todos los ámbitos de la vida (Fernández, Cecchini, \& Zagalaz, 2002). En este aspecto, el Diseño Curricular Base (DCB) de Educación Primaria relaciona el área de Educación Física (EF) con la adquisición de competencias en salud y hábitos saludables de actividad física. Más concretamente, se menciona en los estándares de aprendizaje evaluable de sexto curso "Mejora de la condición física global, conociendo los distintos parámetros y factores que inciden sobre ella a través de formas jugadas". En la literatura científica también se hace hincapié en que el área de educación física es la encargada de trabajar la condición física y su importancia en la vida de los seres humanos (Fernández et al., 2002). Por tanto, el aprendizaje del deporte en las clases de EF puede contribuir al desarrollo de la condición física si se plantea con una metodología adecuada.

El docente es el encargado de transmitir los conocimientos a los alumnos adecuándose a los estándares de aprendizaje evaluable definidos en el DCB en función de la metodología empleada (Feliz \& Ricoy, 2002). La metodología de 
enseñanza-aprendizaje afecta a todas las variables que influyen en la carga soportada por los estudiantes durante la sesión de clase, así como a las variables pedagógicas que definen una tarea, como son la situación de juego, la fase de juego, los medios de iniciación al entrenamiento, tipos de contenido, etc. (Ibáñez, Feu, \& Cañadas, 2016), condicionando las respuestas que realizan los alumnos durante la clase de EF, influyendo en la mejora de la condición física. Los docentes utilizan en el diseño de sus tareas situaciones y medios de iniciación descontextualizados de la práctica del juego real (Feu, García-Rubio, GameroPortillo \& Ibáñez, 2019), condicionando la carga de la tarea. En esta línea, las intensidades de las sesiones de EF deberían cumplir con las recomendaciones de la Organización Mundial de la Salud (OMS) y las evidencias científicas para la mejora de la salud de los estudiantes.

Se puede definir la carga de entrenamiento como "la suma de estímulos a los que el jugador se ve sometido durante el proceso de preparación" (García, Parejo \& Cañadas, 2010). La intensidad y la carga externa (CE) de las sesiones está influenciada por la metodología de enseñanza que el docente utiliza (Bendiksen, et al., 2014). Dentro de las metodologías de enseñanza-aprendizaje existen dos grandes enfoques para la enseñanza de los deportes de invasión: TeacherCentered Approach (TCA) y Student-Centered Approach (SCA). La metodología Direct Instruction (DI) es uno de los métodos típicos del enfoque TCA (Metzler, 2011). En la metodología TCA el profesor tiene el control absoluto en las sesiones de enseñanza. El docente decide el contenido a desarrollar, la gestión de la clase y la participación de los estudiantes. Las tareas están diseñadas para crear patrones de movimiento y habilidades técnicas en los estudiantes (Metzler, 2011). El medio de enseñanza para la iniciación más habitual en las tareas de esta metodología son los ejercicios de aplicación simple e inespecífico (Alarcón, Cárdenas, Miranda, Ureña, \& Piñar, 2011; Cañadas, Ibáñez, Feu, García-Rubio, \& Parejo, 2011; Cañadas, Ibáñez, Feu, García, \& Parejo, 2011). La progresión de las habilidades técnicas se inicia con un modelo visual expuesto por el profesor, un alumno o en vídeo que los estudiantes deben repetir. En primer lugar, se practica una habilidad técnica de forma descontextualizada del juego real para posteriormente trabajarla en una tarea dentro del juego (Pill, 2015).

Por otro lado, en el enfoque SCA, el método Tactical Game Approach (TGA) es una de las metodologías que pueden utilizarse en el contexto escolar (Mitchell, Oslin, \& Griffin, 1997). Esta metodología aumenta la complejidad táctica dividiendo este proceso en tres etapas: el juego modificado, el desarrollo de la conciencia táctica y la toma de decisiones. Las tareas se basan en formas de juego variadas donde hay que resolver un problema táctico (Mitchell et al., 1997). Según Mitchell et al., (1997) la clave para que los alumnos resuelvan los problemas tácticos son los feedbacks que los docentes utilizan para orientar al alumno a que consiga el objetivo propuesto. Esta metodología se basa en ejercicios de aplicación complejos y en juegos complejos específicos (Cañadas et al., 2011). A pesar de los beneficios pedagógicos y motivacionales que los TGA tienen sobre los alumnos, algunos autores ponen en duda la intensidad de la práctica en estos métodos (Chen, Martin, Sun, \& Ennis, 2007).

Diversos estudios se han centrado en comparar metodologías de enseñanzaaprendizaje (González-Espinosa, Feu, García-Rubio, Antúnez, \& García-Santos, 
2017; Harvey, Cushion, Wegis, \& Massa-Gonzalez, 2010; Mesquita, Farias, \& Hastie, 2012). Según Miller (2015) los estudios que comparan metodologías de enseñanza han tenido como objetivo el comparar variables como habilidad técnica, conocimiento del deporte, rendimiento en el juego y variables afectivas. Se han encontrado diferencias en las variables pedagógicas y de rendimiento a favor de las metodologías del enfoque SCA. Los estudios que analizan variables de la carga de la tarea en la enseñanza de los deportes de invasión en el contexto escolar son escasos aún. Esto puede deberse a la escasez de recursos materiales que los docentes disponen para sus sesiones de EF que les permitan cuantificar la carga de las tareas y de las sesiones de entrenamiento.

Para cuantificar la carga de las sesiones de EF a través de la CE, pueden emplearse diferentes instrumentos que ayuden a los docentes a cuantificarla de forma objetiva o subjetiva. Los métodos subjetivos sustituyen la falta de tecnología o el difícil acceso a la misma. Una herramienta subjetiva muy empleada para medir la CE es la valoración subjetiva del esfuerzo (RPE) durante una sesión de enseñanza (Borresen \& Lambert, 2008; Foster et al., 2001). Ibáñez, Feu y Cañadas (2016) proponen un sistema integral para el análisis de las tareas (SIATE). EI SIATE es una herramienta de observación sistemática (Morgan, Muir, \& Abraham, 2014) que permite cuantificar mediante un sistema de categorías la carga externa, además de analizar variables pedagógicas y organizativas. Dentro de los instrumentos que miden de forma objetiva la CE han aparecido en los últimos años los sistemas inerciales de medición del movimiento equipados con múltiples sensores, tales como acelerómetros o los sistemas de global positioning systems (GPS). Esta herramienta permite medir la CE, registrando las distancias, velocidades o aceleraciones (Delextrat et al., 2015; O'Hara et al., 2013), con una gran una alta fiabilidad y validez (Barrett, Midgley, \& Lovell, 2014; Beato, Bartolini, Ghia, \& Zamparo, 2016; Muñoz-Lopez, GraneroGil, Pino-Ortega, \& De Hoyo, 2017).

Reina, Mancha, García-Santos, García-Rubio \& Ibáñez (2019) realizaron un estudio comparativo sobre la carga de entrenamiento soportada por jóvenes jugadores de baloncesto, a través de tres tipos de instrumentos, dispositivos inerciales (carga externa objetiva), bandas de frecuencia cardíaca (carga interna objetiva) y la hoja de registro de entrenamiento propuesta por el SIATE (carga externa subjetiva). Los resultados mostraron una alta correlación entre los tres instrumentos de medida. En esta misma línea, Gómez-Carmona, Gamonales, Feu, \& Ibáñez (2019) también encontraron una alta correlación entre carga externa objetiva medida a través de dispositivos inerciales y la carga externa subjetiva, medida a través del SIATE, en las tareas de un equipo de fútbol. Estos datos ponen de manifiesto la importancia del registro de la carga de entrenamiento, con independencia de los medios empleados para ello, pues ya sean costosos y tecnológicos o básicos, reportan información equivalente.

El estudio de la CE está muy extendido en el estudio de los deportes de equipo como el baloncesto (Delextrat et al., 2015; Reina, Mancha, \& Ibáñez, 2017; Torres-Ronda, Ric, Llabres-Torres, de Las Heras, \& Schelling, 2016), pero hasta donde se conoce, son escasos los estudios que analicen la CE las sesiones de EF. 
El desarrollo de las sesiones de EF implica una carga de entrenamiento a los alumnos y esa carga está condicionada por la metodología que el docente utiliza en las sesiones. Las diferentes metodologías de enseñanza-aprendizaje tienen un diseño de tareas diferentes en variables como: situación de juego, fase de juego, tipo de contenido, contenido específico, medio de iniciación al entrenamiento y nivel de oposición (González-Espinosa, Ibáñez \& Feu, 2017). El diseño de las tareas está condicionado con el medio de iniciación al entrenamiento y la situación de juego. Según el medio de iniciación al entrenamiento y la situación de juego, la intensidad de las tareas puede variar. Según las recomendaciones de la OMS (2010) y diferentes estudios científicos sobre las recomendaciones en la intensidad en las actividades físico deportivas en la edad escolar es preciso que los alumnos tengan una intensidad alta en sus sesiones de EF (Bendiksen et al., 2014; Ingul, Tjonna, Stolen, Stoylen, \& Wisloff, 2010; Sperlich et al., 2010). Dada la escasez de estudios que comparen la CE de las tareas que utilizan los docentes en el contexto escolar y su metodología, hacen necesaria la realización de estudios que aporten claridad sobre las demandas físicas que plantean las tareas para el aprendizaje de los deportes en la escuela. Los nuevos dispositivos inerciales permiten cuantificar la CE de forma objetiva. Por todo lo anterior, el objetivo de este estudio es caracterizar y comparar la CE de los diferentes tipos de tareas que se desarrollan en las sesiones de EF entre dos metodologías de enseñanza-aprendizaje diferentes, en la enseñanza de un deporte de colaboración-oposición como el baloncesto.

\section{MÉTODO}

\section{Diseño}

El diseño del presente estudio fue definido como cuasi experimental ya que pretende establecer una relación causa-efecto y cumplen con el requisito de la manipulación de al menos una variable independiente, pero no es posible (o no es ético) cumplir con la asignación aleatoria para asegurar que no hay diferencias entre grupos antes de asignar un programa a cada uno. Además, está dentro de los estudios longitudinales de medidas repetidas (pretest y postest) (Ato, López y Benavente, 2013).

\section{Muestra}

La muestra del estudio estaba compuesta por un total de 40 tareas, 20 en cada metodología de enseñanza. La unidad básica de análisis fue el registro de cada alumno en cada tipo de tarea. Se registraron un total de 2139 registros para cada variable del estudio. De estos registros, 1087 pertenecen al programa basado en la metodología TGA, TGB, y 1052 al programa basado en la metodología DI, DIB. En la Tabla 1 se muestran la totalidad de registros recogidos en función de las situaciones de juego que pertenecen a los programas de intervención. 
Tabla 1. Distribución de la muestra según la situación de juego y la metodología de enseñanza-aprendizaje.

\begin{tabular}{lccc}
\hline & TGB & DIB & TOTAL \\
\hline 1vs0 & 488 & 425 & 913 \\
Off +1 & 46 & 89 & 135 \\
1vs1 & 278 & 253 & 531 \\
2 vs2 & 177 & 194 & 371 \\
3vs3 & 98 & 91 & 189 \\
TOTAL & 1087 & 1052 & 2139 \\
\hline
\end{tabular}

TGB: Programa de intervención Tactical Game in Basketball. DIB: Programa de intervención Direct Instruction in Basketball. Off+1: situación de juego con superioridad ofensiva de un jugador

\section{Participantes}

Para el desarrollo de estas tareas participaron 70 alumnos de entre 10 y 12 años $(M=10.86+/-0.53)$ de un centro de educación infantil y primaria en el suroeste de España. Los alumnos pertenecen a dos niveles educativos, $5^{\circ}$ de primaria y 6 o de primaria. En cada nivel educativo tenía dos grupos por lo que se contaron con cuatro grupos en total: 5ㅅ (13 alumnos), 5ํ B (12 alumnos), 6ำ (21 alumnos) y $6^{\circ} \mathrm{B}$ (24 alumnos). Los alumnos de $5^{\circ} \mathrm{A}$ y $6^{\circ} \mathrm{A}$ fueron los que recibieron el programa de intervención Tactical Game in Basketball (TGB). Con los alumnos de las clases 5B y 6으 se desarrolló el programa de intervención Direct Instruction in Basketball (DIB). Se han formado al azar por parte del centro educativo desde que los alumnos entran a formar parte del centro escolar (3 años). La elección de los diferentes programas para cada grupo se hizo de forma azarosa y antes de conocer a los alumnos. Todos los alumnos de ambos grupos llevan un dispositivo inercial de registro de actividad física.

\section{Declaración ética}

El centro educativo autorizó la realización de este estudio, añadiendo a la programación general anual la intervención realizada con los alumnos. Todos los padres/tutores de los alumnos firmaron el consentimiento informado para autorizar la participación de los estudiantes en el estudio. La investigación cumple con los principios éticos en trabajos de investigación de la Declaración de Helsinki y fue aprobada previamente por el comité de ética de la Universidad local (no 67/2017).

\section{Variables}

\section{Programas de enseñanza}

Los dos programas de intervención Direct Instrucction in Basketball (DIB), basado en la metodología de DI, y el programa Tactical Game in Basketball (TGB), basado en la metodología TGA, han sido validados (González-Espinosa, et al., 2017) y diseñados (González-Espinosa, et al., 2017) antes de la intervención en este estudio. 
El DIB y el TGB tienen los mismos objetivos didácticos que cumplir: descubrir los medios técnico-tácticos que permiten desplazarse por el terreno de juego para lanzar a canasta, experimentar el lanzamiento a canasta, presentar los conceptos básicos de la fase defensiva e iniciar al juego colectivo. Para cumplir estos objetivos se diseñaron 20 tareas para cada metodología de enseñanzaaprendizaje (Tabla 2). La situación de juego de las tareas del DIB y el TGB eran 1vs0, 1vs1, 2vs2, 3vs2 y 3vs3. Con estas situaciones de juego se pretende conseguir el objetivo de área que es la iniciación al deporte.

Tabla 2. Objetivos de los programas de intervención.

\begin{tabular}{clc}
\hline DIB & \multicolumn{1}{c}{ OBJETIVO } & TGB \\
\hline & $\begin{array}{l}\text { Descubrir los medios técnico- } \\
\text { tácticos que permiten } \\
\text { desplazarse por el terreno de } \\
\text { juego para lanzar a canasta. }\end{array}$ & A1, A2, A10, A13 \\
\hline $\begin{array}{c}\text { T2, T10, T13 } \\
\text { T4, T5, T6, T7, T8, T9, } \\
\text { T11 }\end{array}$ & $\begin{array}{l}\text { Experimentar el lanzamiento } \\
\text { a canasta. }\end{array}$ & $\begin{array}{c}\text { A3, A4, A5, A6, A7, A8, A9, } \\
\text { A11 }\end{array}$ \\
\hline T12, T19 & $\begin{array}{l}\text { Presentar los conceptos } \\
\text { básicos de la fase defensiva. }\end{array}$ & A12, A19 \\
\hline T14, T15, T16, T17, T18, & Iniciar al juego colectivo & A14, A15, A16, A17, A18, \\
T20 & & A20 \\
\hline
\end{tabular}

Las 20 tareas se desarrollan durante diez sesiones de intervención. Los programas de intervención se analizaron a través del SIATE, identificando que en las variables pedagógicas que definen las tareas de entrenamiento, no existían diferencias. Se analizó la fase de juego (fase en la que se encuentra un equipo en deportes invasión la determina, habitualmente, la posesión del balón), tipo de contenido (se agrupan en contenidos individuales, grupales y de equipo, tanto para el desarrollo de los contenidos de la fase de ataque y defensa, así como para las conductas tácticas y gestos técnicos), contenido específico (contenido del deporte específico a trabajar) y espacio de juego (espacio en el que los deportistas tienen que realizar las tareas propuestas).

La duración de cada programa de intervención es de un total de diez sesiones. Las diez sesiones se dividieron en dos partes: las cinco sesiones iniciales estaban focalizadas hacia el juego individual y las cinco sesiones finales hacia el juego colectivo. Las situaciones de juego aumentan con la evolución de las sesiones, empezando por situaciones de juego simples (1vs0 y 1vs1), para acabar en situaciones próximas al juego real (3vs3). Las sesiones estaban divididas en cinco tareas cada una, aumentando la complejidad de la situación de juego de forma progresiva. Cada sesión tenía una duración de 50 minutos, utilizando los 10 minutos iniciales para colocar los dispositivos inerciales, teniendo un tiempo total de la sesión de 40 minutos.

En el contexto escolar, el currículum oficial propone en el área de educación física en el bloque 2: deporte, juegos y salud como contenido a trabajar el deporte en la iniciación. Se consideró por parte de los investigadores que la situación de juego más compleja a trabajar en el contexto escolar fuera el 3vs3. Esta situación de juego cumple con los principios técnico-tácticos del deporte del baloncesto en la iniciación y es una forma de juego reducido mejor que la del 5vs5 (McCormick et al., 2012). Además, la situación de juego 3vs3 es una adaptación que se 
adaptada a las necesidades del currículum oficial. Para llegar a la situación de juego 3vs3, se trabajan situaciones de juego previas para conseguir progresar en complejidad y situación del juego hasta el 3vs3 como propone lbáñez (2002).

\section{Situación de juego}

Se entiende por situación de juego a las agrupaciones de estudiantes que los docentes diseñan para cada una de las tareas (Ibáñez et al., 2016). Las diferentes situaciones de juego empleadas para esta investigación han sido: a) un atacante sin oposición (1vs0), b) un atacante contra un defensor (1vs1), c) dos atacantes contra un defensor o tres atacantes contra dos defensores, 2vs1 y $3 v s 2,(\mathrm{Off}+1)$, e) dos atacantes contra dos defensores (2vs2) y e) tres atacantes contra tres defensores (3vs3).

Las situaciones de juego que se trabajan en ambos métodos son similares debido a que todas las tareas diseñadas partían del mismo objetivo. A partir del objetivo a trabajar se diseñaban dos tareas análogas, una para cada programa, con una situación de juego semejante en ambas metodologías. Las diferencias en la situación de juego son debido a que la metodología TGA utiliza situaciones de juego próximas al juego real (igualdades numéricas) y la metodología DI utiliza situaciones de juego sin defensa y descontextualizadas.

\section{Variables de CE}

Las variables de CE cinemáticas que se utilizaron en el trabajo fueron: i) metros recorridos por minuto $(\mathrm{m} / \mathrm{min})$;ii) aceleraciones por minuto, cambios en la magnitud de velocidad de más de $+2 \mathrm{~m} / \mathrm{s}^{2}(\mathrm{acc} / \mathrm{min})$; iii) desaceleraciones por minuto, cambios en la magnitud de velocidad de más de $-2 \mathrm{~m} / \mathrm{s}^{2}$ (dece $\left./ \mathrm{min}\right)$; iv) velocidad máxima $\left(\mathrm{V}_{\text {máx }}\right)$; v) velocidad media $(\mathrm{V})$; vi) actividad de alta intensidad (HIA) (actividades desarrolladas a más de $16 \mathrm{~km} / \mathrm{h}$ durante un tiempo superior a 5 segundos); vii) walk (<6 km/h); viii) jog $(6-12 \mathrm{~km} / \mathrm{h})$; ix) run $(12-18 \mathrm{~km} / \mathrm{h}) ; \mathrm{x}$ ) sprint $(>18 \mathrm{~km} / \mathrm{h})$; xi) número de sprints $\left(\mathrm{N}_{\text {sprints }}\right)$; $\left.x \mathrm{ii}\right)$ pasos por minuto (steps/min); xiii) saltos por minuto( $>3 G$ y $>600 \mathrm{~ms}$ ) (jumps/min). Las variables de CE neuromusculares fueron: Player Load máximo $\left(P L_{\text {máx }}\right)$ y Player Load por minuto (PL/min). El PL es la suma de las aceleraciones producidas en los tres planos del movimiento, lo que estima la carga de entrenamiento total (Barrett et al., 2014).

Las variables de velocidad del estudio se han adaptado para la muestra. Para ello, se realizó un cluster de K-medias para 4 grupos. El análisis estadístico identificó cuatro rangos de velocidades que fueron caracterizadas como walk (0$6 \mathrm{~km} / \mathrm{h})$, jog (6-12 km/h), run (12-18 km/h) and sprint (> $18 \mathrm{~km} / \mathrm{h})$. El N $\mathrm{N}_{\text {sprint }}$ se considera cada vez que la velocidad superaba los $18 \mathrm{~km} / \mathrm{h}$.

\section{Instrumentos}

Para el registro de las variables de CE se utilizaron los dispositivos inerciales Wimu $^{\mathrm{TM}}$ (RealTrack Systems ${ }^{\mathrm{TM}}$ ). Estos dispositivos son herramientas de registro inercial de monitorización de la actividad física y el movimiento a tiempo real. 
Esta herramienta registra los datos a través de ocho sensores diferentes que obtienen datos para cada uno de los sensores (acelerómetros, podómetros, GPS, etc.). Los dispositivos se ajustan a los sujetos a través de unos chalecos en donde se deposita el Wimu ${ }^{\mathrm{TM}}$. Los sensores aportan un archivo con los datos puros de cada sensor para posteriormente hacer la combinación de ellos y sacar todas las variables posibles de los mismos. Este registro se analiza con el software oficial Spro ${ }^{\mathrm{TM}}$ (RealTrack Systems ${ }^{\mathrm{TM}}$ ).

\section{Procedimiento}

Antes de la intervención en el contexto escolar, se realizó una formación sobre la intervención en ambas metodologías de enseñanza aprendizaje con el docente que iba a llevar a cabo las sesiones con ambos programas de enseñanza. El docente que realizó las sesiones es graduado en educación y con formación investigadora en metodologías de enseñanza. Se trata de un investigador formado exprofeso para la aplicación de los programas. Para ello, tuvo un conocimiento profundo de las características de la investigación, de los programas y de la forma de intervenir en cada uno de ellos, evitando el sesgo. Previo al desarrollo de la investigación se realizaron sesiones prácticas piloto con otro grupo de estudiantes para comprobar la idoneidad de la intervención. Además, para controlar al docente durante las sesiones se registró con un micrófono y vídeo su intervención.

En primer lugar, se procedió a la recogida de datos en la escuela. Para ello se aplicó el programa DIB en dos grupos y el programa TGB en otros dos grupos como se ha explicado en los participantes del estudio. Todas las sesiones fueron registradas con los dispositivos Wimu $^{\mathrm{TM}}$.

Tras la intervención en la escuela, se procedió con el análisis de los datos obtenidos en las sesiones de enseñanza. Para el tratamiento y análisis de los datos obtenidos con los dispositivos inerciales se utilizó el software Spro ${ }^{\mathrm{TM}}$. Se realizó un análisis exploratorio para eliminar los outliers del conjunto de datos. A continuación, se utilizó un análisis Cluster de K-means para establecer los rangos de las variables relacionadas con la velocidad, adaptándolos y personalizándolos a la muestra del estudio. Con este conjunto de datos finales se elaboró en el Spro ${ }^{\mathrm{TM}}$ una configuración de análisis específica para la muestra del estudio. Con esta configuración se realizaron los diferentes análisis de la investigación para obtener los resultados finales.

\section{Análisis de datos}

Se normalizaron todas las variables de CE a la unidad por minuto para poder comparar adecuadamente cada registro de datos. En primer lugar, se realizaron las pruebas de asunción de criterios: normalidad (Kolmogorov-Smirnov), homocedasticidad (Levene) y aleatoriedad (Rachas) (Field, 2009). Con los resultados obtenidos en las pruebas de asunción de criterios se determinó el uso de pruebas no paramétricas para el contraste de las hipótesis. A continuación, se realizó un análisis descriptivo, a través de las medias y la desviación típica de todas las variables. Para comparar las diferentes situaciones de juegos en el 
programa DIB y la TGB se empleó la prueba $U$ de Mann-Whitney (Field, 2009). Para analizar las diferencias entre los tipos de tareas para cada una de las metodologías se realizó la prueba estadística $H$ de Kruskal-Wallis. Además, se calculó el tamaño del efecto para conocer la relevancia de las diferencias encontradas entre los dos grupos comparados. El tamaño del efecto fue calculado por Cohen's $d$ from F-test. Se consideran que los tamaños del efecto son pequeños cuando el valor es .20, medio con un valor de .50 , y grande con un valor de .80 (Thalheimer \& Cook, 2002). Para el análisis estadístico se utilizó el software SPSS v.21 (IBM SPSS Statistics para Windows, Versión 21.0. Armonk, NY: IBM Corp). El valor de la significación utilizado en este estudio fue $p<.05$.

\section{RESULTADOS}

En primer lugar, se muestran los resultados descriptivos del programa DIB y TGB. La Figura 1 muestra los resultados descriptivos de cada variable analizada para la situación de juego de $1 \mathrm{vs} 0$.

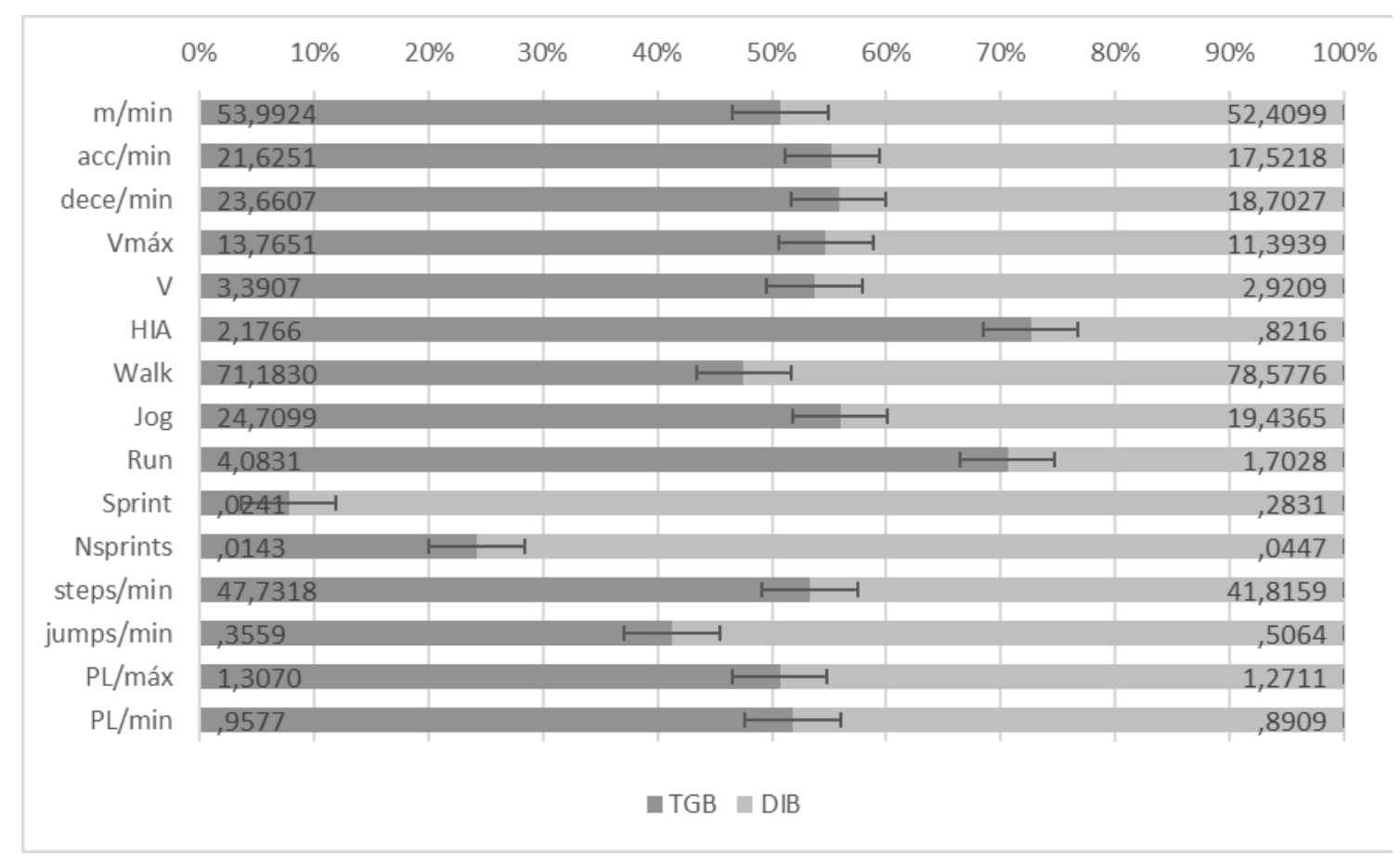

Figura 1. Resultados descriptivos de la situación de juego 1vs0

En la Figura 1 se presenta la media de cada grupo con número y las barras de colores representan el peso de la media frente a la media del otro grupo. Además, se presenta la desviación de ambas medidas.

Los resultados muestran cómo en la situación de juego $1 v s 0$ el programa TGB tienen valores más altos en $\mathrm{m} / \mathrm{min}$, acc/min, dece/min, $V_{\text {máx, }} V$, HIA, jog, run, steps/min, $P L_{\text {máx y }} \mathrm{PL} / \mathrm{min}$. El programa DIB tiene valores más altos en las variables walk, sprint, $N_{\text {sprint }} y$ jumps/min. Las variables HIA ( $\mathrm{TGB}=2.177 / \mathrm{DIB}=.822), \quad$ run $\quad$ ( $\mathrm{TGB}=4.083 / \mathrm{DIB}=1.703), \quad$ sprint (TGB=.024/DIB=.283) y $N_{\text {sprint }}(T G B=.014 / \mathrm{DIB}=.045)$ son las que más diferencias 
tienen en los estadísticos descriptivos entre los dos programas de intervención aplicados.

En la Figura 2 se observan los resultados descriptivos de cada variable analizada en la situación Off + 1 .

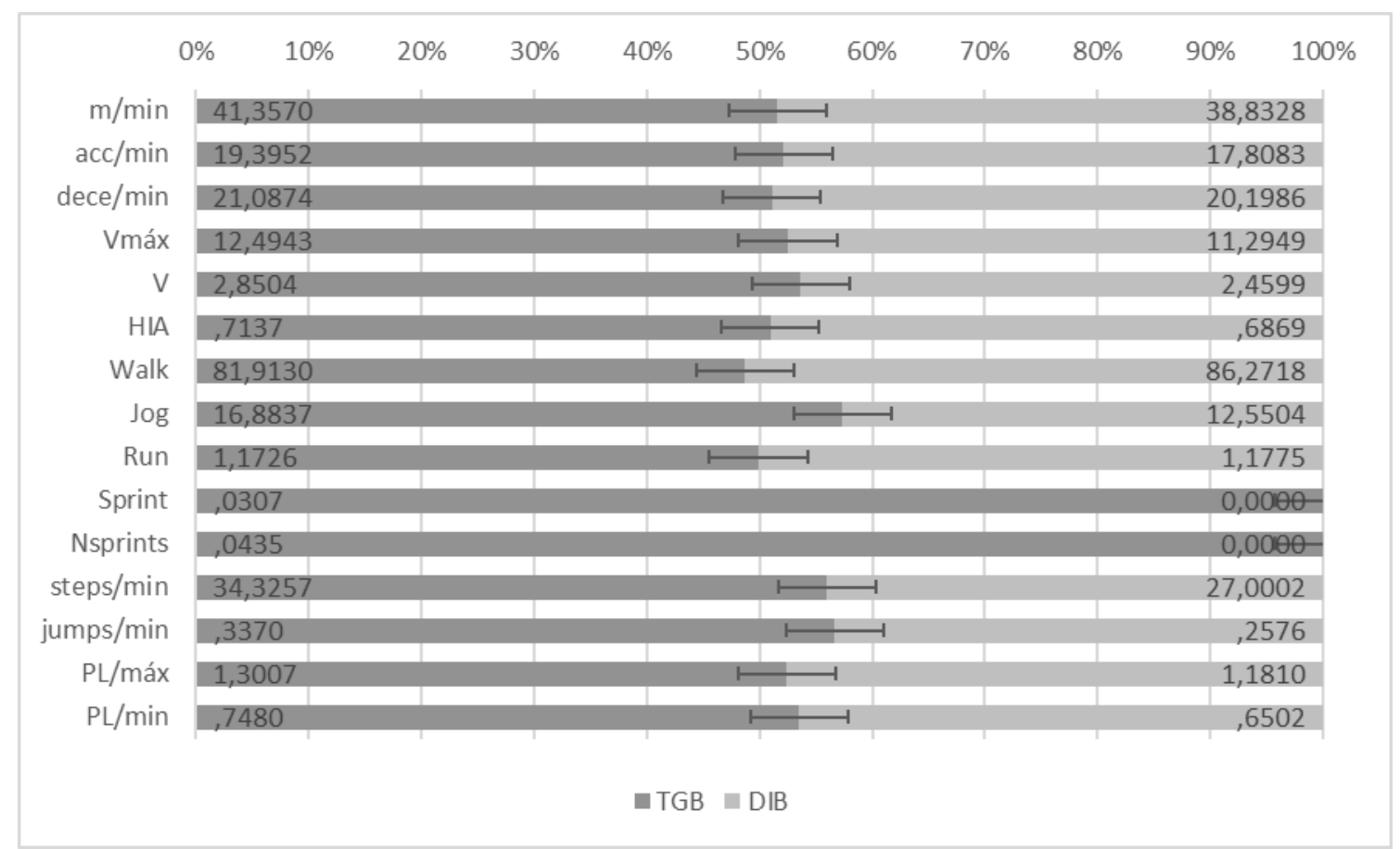

Figura 2. Resultados descriptivos en el contenido Off +1 .

En tareas cuya situación de juego son las superioridades ofensivas de un jugador destaca que todas las variables, excepto walk $(T G B=81.91 / D I B=86.27)$ y run $(\mathrm{TGB}=1.173 / \mathrm{DIB}=1.178)$ obtienen valores más altos en el programa TGB. Entre todas las variables, destaca que en el DIB no hay velocidades de sprint y por lo tanto tampoco hay ningún sprint registrado dado que el tipo de tareas propias de la metodología DI no requiere que los alumnos tengan una velocidad máxima.

En la Figura 3 se presentan los resultados descriptivos de la situación de juego 1 vs1 en función de la metodología de enseñanza-aprendizaje utilizada. 
Rev.int.med.cienc.act.fís.deporte - vol. 20 - número 79 - ISSN: 1577-0354

\begin{tabular}{|c|c|c|c|c|c|c|c|c|c|}
\hline & $10 \%$ & $20 \%$ & $30 \%$ & $40 \%$ & $50 \%$ & $60 \%$ & $70 \%$ & $80 \%$ & $90 \%$ \\
\hline $\mathrm{m} / \mathrm{min}$ & 48,6640 & & & & 1 & & & & 50,4985 \\
\hline $\mathrm{acc} / \mathrm{min}$ & 21,4530 & & & 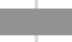 & $\mapsto$ & & & & 19,8001 \\
\hline dece/min & 23,3004 & & & 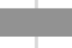 & $\mapsto$ & & & & 21,8982 \\
\hline Vmáx & 12,1039 & & & 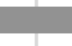 & F & & & & 11,1262 \\
\hline V & 2,9266 & & & 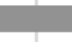 & F & & & & 2,6882 \\
\hline HIA &, 7205 & & & 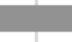 & $\mapsto$ & & & . &, 6624 \\
\hline Walk & 80,8210 & & & 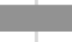 & $\mapsto$ & & & & 83,9593 \\
\hline Jog & 17,8303 & & & 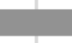 & 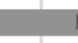 & & 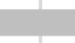 & 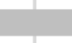 & 14,7685 \\
\hline Run & 1,1954 & & & 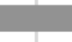 & $\mapsto$ & & 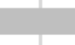 & 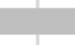 & 1,1419 \\
\hline Sprint & , 1529 & & & 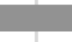 & $r$ & & 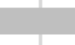 & 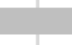 & ,1306 \\
\hline Nsprints &, 0287 & & & 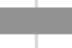 & & 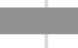 & & &, 0154 \\
\hline steps/min & 38,7902 & & & 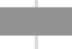 & $\mapsto$ & & & & 37,5981 \\
\hline jumps/min & , 2766 & & & 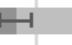 & & & & 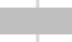 & ,4480 \\
\hline PL/máx & 1,1150 & & & & -1 & & & 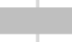 & 1,1428 \\
\hline $\mathrm{PL} / \mathrm{min}$ & ,7932 & & & & $\mapsto$ & & & & ,8161 \\
\hline \multicolumn{10}{|c|}{$\square \mathrm{TGB}=\mathrm{DIB}$} \\
\hline
\end{tabular}

Figura 3. Resultados descriptivos en el contenido de 1vs1

En el análisis descriptivo de la variable 1vs1 existe igualdad en casi todas las variables del estudio entre ambos métodos, aunque la mayoría obtiene valores levemente superiores en el programa TGB. Las variables que destacadan son $N_{\text {sprint }}(\mathrm{TGB}=.029 / \mathrm{DIB}=.015)$ y jumps $/ \mathrm{min}(\mathrm{TGB}=.277 / \mathrm{DIB}=.448)$.

La Figura 4 recoge los resultados de la situación de juego de 2vs2 en cada uno de los programas de intervención realizados.

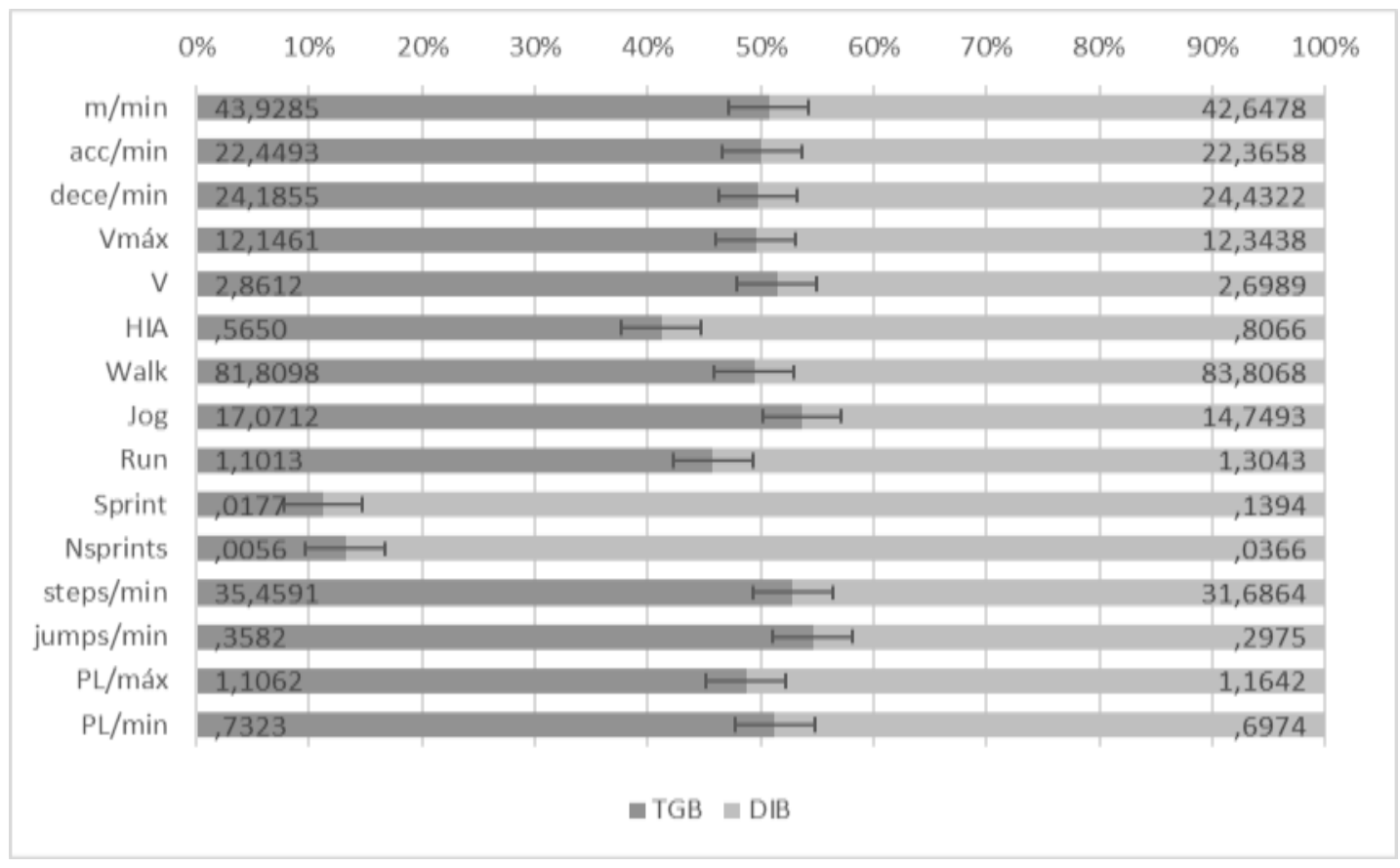

Figura 4. Resultados descriptivos en el contenido de 2vs2. 
En la situación de juego 2vs2 se observa cómo el número de diferencias entre ambos métodos de enseñanza se neutraliza excepto en las variables $H I A$ (TGB=.565/DIB=.807), sprint $\quad(\mathrm{TGB}=.0178 / \mathrm{DIB}=.139)$ y $\quad N_{\text {sprint }}$ ( $\mathrm{TGB}=.006 / \mathrm{DIB}=.037$ ) en donde el método $\mathrm{DIB}$ obtiene valores más altos que el TGB.

La Figura 5 muestra los valores medios y desviación típica obtenidos por ambas metodologías en la situación de juego 3vs3.

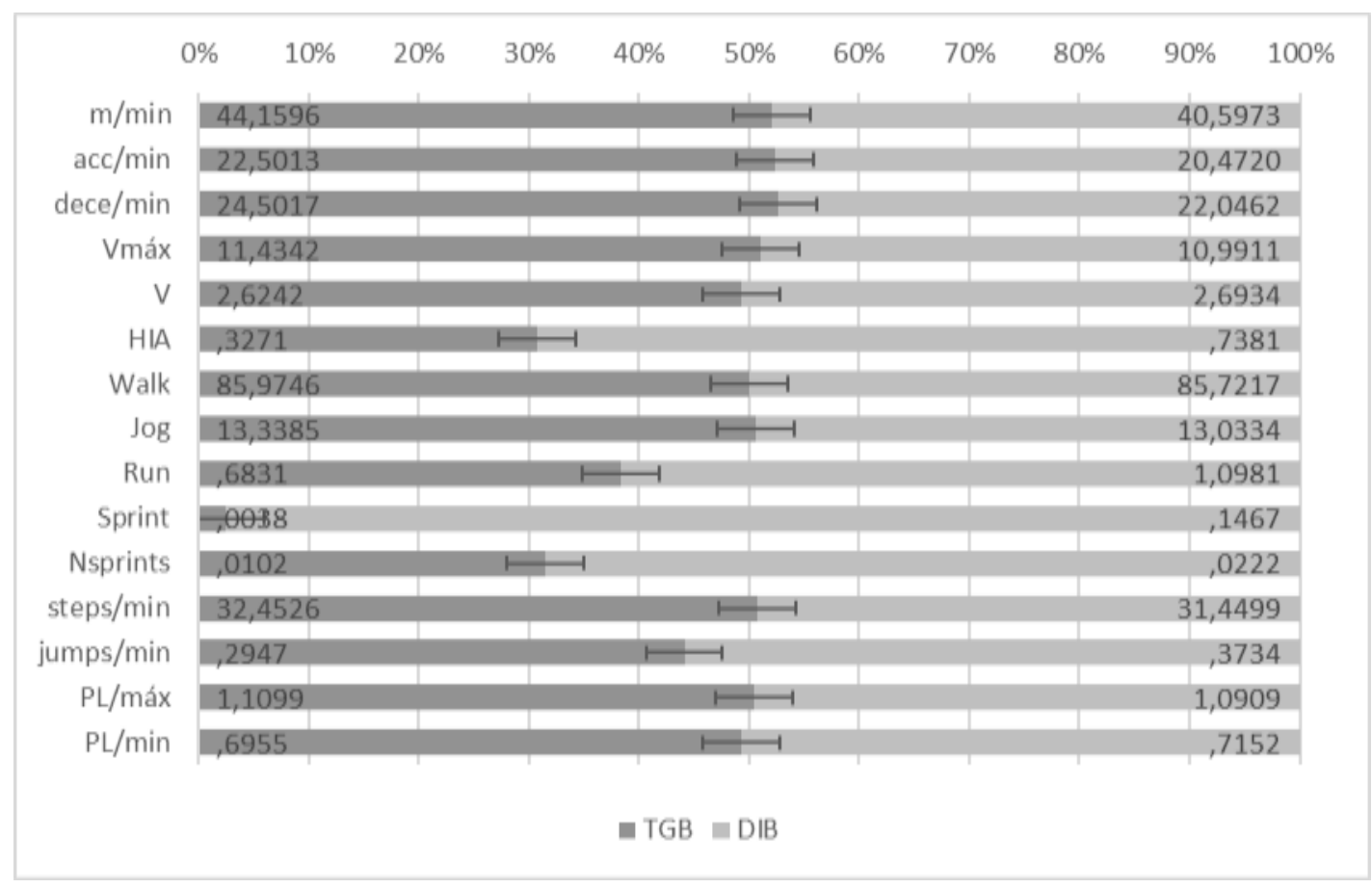

Figura 5. Resultados descriptivos en el contenido de 3vs3.

Las variables donde existen mayores diferencias entre los métodos de enseñanza son HIA (TGB =.327/DIB =.738), run (TGB =.683/DIB =1.098), sprint $(\mathrm{TGB}=.004 / \mathrm{DIB}=.147)$ y $N_{\text {sprint }}(\mathrm{TGB}=.010 / \mathrm{DIB}=.022)$. Todas estas variables tienen valores más altos en el programa DIB. En el resto de variables existen diferencias entre ambos programas, pero no son significativas.

En la Tabla 3 se muestran las diferencias entre de los programas de intervención en todas las variables de CE analizadas en función de la situación de juego. 
Tabla 3. Comparación de las situaciones de juego en función del método de enseñanza.

\begin{tabular}{|c|c|c|c|c|c|c|c|c|c|c|c|c|c|c|c|}
\hline & $1 \mathrm{vs} 0$ & $\eta^{2}$ & $d$ & Off +1 & $\eta^{2}$ & $d$ & 1vs1 & $\eta^{2}$ & $d$ & 2vs2 & $\eta^{2}$ & $d$ & 3vs3 & $\eta^{2}$ & $d$ \\
\hline $\mathrm{M} / \mathrm{min}$ & $.000^{* *}$ & .021 & .296 & .220 & .011 & .212 & .744 & .000 & .028 & .246 & .004 & .121 & .071 & .017 & .265 \\
\hline Acc/min & $.000^{* *}$ & .023 & .306 & .219 & .011 & .213 & .194 & .003 & .111 & .513 & .001 & .068 & .403 & .004 & .122 \\
\hline Dece/min & $.000^{\star \star}$ & .037 & .390 & .334 & .007 & .166 & .218 & .003 & .106 & .542 & .001 & .063 & .359 & .004 & .134 \\
\hline$V_{\text {máx }}$ & $.000^{* *}$ & .188 & .962 & $.030^{*}$ & .035 & .380 & $.000^{\star *}$ & .028 & .342 & .562 & .001 & .060 & .057 & .019 & .280 \\
\hline V & $.000^{* *}$ & .145 & .824 & $.000^{\star *}$ & .104 & .681 & $.000^{\star *}$ & .054 & .478 & $.000^{* *}$ & .039 & .401 & .428 & .003 & .116 \\
\hline HIA & $.000^{* *}$ & .143 & .817 & .194 & .008 & .180 & .059 & .004 & .134 & .580 & .001 & .048 & .585 & .001 & .057 \\
\hline Walk & $.000^{* *}$ & .093 & .642 & $.014^{*}$ & .045 & .433 & $.000^{* *}$ & .031 & .357 & .013 & .017 & .259 & .857 & .000 & .026 \\
\hline Jog & $.000^{* *}$ & .065 & .527 & $.003^{* *}$ & .065 & .526 & $.000^{* *}$ & .030 & .351 & .002 ** & .025 & .320 & .747 & .001 & .047 \\
\hline Run & $.000^{* *}$ & .198 & .932 & .208 & .010 & .197 & $.003^{* *}$ & .014 & .241 & .448 & .001 & .074 & .431 & .002 & .099 \\
\hline Sprint & .366 & .000 & .022 & $.048^{*}$ & .001 & .071 & .548 & .000 & .016 & .061 & .001 & .062 & .267 & .000 & .041 \\
\hline$N_{\text {sprints }}$ & $.021^{*}$ & .000 & .041 & $.048^{*}$ & .001 & .071 & .246 & .000 & .023 & .117 & .000 & .036 & .512 & .000 & .021 \\
\hline Steps/min & $.000^{* *}$ & .037 & .390 & $.022^{*}$ & .039 & .401 & .407 & .001 & .071 & $.035 *$ & .012 & .219 & .548 & .002 & .088 \\
\hline Jumps/min & $.000^{\star *}$ & .018 & .274 & $.043^{*}$ & .030 & .349 & $.000^{\star \star}$ & .045 & .436 & .378 & .002 & .090 & .857 & .000 & .026 \\
\hline PLmáx & .297 & .001 & .068 & .413 & .005 & .141 & .233 & .003 & .102 & .268 & .003 & .115 & .610 & .001 & .074 \\
\hline $\mathrm{PL} / \mathrm{min}$ & $.001^{* *}$ & .011 & .216 & .095 & .020 & .289 & .219 & .003 & .105 & .204 & .004 & .132 & .779 & .000 & .041 \\
\hline
\end{tabular}

En el caso de la situación de juego $1 v s 0$ se encuentran diferencias significativas en todas las variables excepto en sprint y $P L_{\text {maxx. }}$ Las situaciones Off +1 tienen más de la mitad de las variables que diferencien el TGB del DIB. En las situaciones de 1vs1 y 2vs2 disminuye el número de variables con diferencias estadísticamente significativas siendo cinco y tres variables las que tienen $p<.05$. En la situación de 3vs3 no hay ninguna variable cuya significación sea estadísticamente significativa para diferencia ambos programas. Los tamaños del efecto muestran diferencias importantes entre metodologías en las situaciones $1 \mathrm{vsO}$ y Off +1 .

En la Tabla 4 se muestran las diferencias entre las situaciones de juego en todas las variables de $\mathrm{CE}$ analizadas en el programa TGB. Se encuentran diferencias significativas en las variables $\mathrm{m} / \mathrm{min}$, $V_{\text {máx }}, V, H I A$, walk, jog, run, steps/min, jumps/min, $P L_{\text {máx }}$ y $P L / m i n$. Las tareas que trabajan las $1 \mathrm{vs} 0$ son las que más se diferencian del resto de tareas. El tamaño del efecto en las variables significativas es medio y grande. 
Tabla 4. Diferencias, tamaño del efecto y potencia entre las diferentes situaciones de juego en el programa de enseñanza TGB.

\begin{tabular}{lllll}
\hline & $p$ & Post-Hoc & $\eta^{2}$ & $d$ \\
\hline M/min & $.000^{* *}$ & A,B,C,D,E,F,G & .115 & $\mathbf{0 . 7 2 4}$ \\
Acc/min & .596 & & & \\
Dece/min & .199 & & & \\
Vmáx & $.000^{* *}$ & A,B,C,D & .141 & $\mathbf{0 . 8 1 1}$ \\
V & $.000^{* *}$ & A,B,C,D,F,H & .233 & $\mathbf{1 . 1 0 2}$ \\
HIA & $.000^{* *}$ & A,B,C,D & .158 & $\mathbf{0 . 8 6 7}$ \\
Walk & $.000^{* *}$ & A,B,C,D,F,H & .265 & $\mathbf{1 . 2 0 1}$ \\
Jog & $.000^{* *}$ & A,B,C,D,F,H & .219 & $\mathbf{1 . 0 6 0}$ \\
Run & $.000^{* *}$ & A,B,C,D & .184 & $\mathbf{0 . 9 4 8}$ \\
Sprint & .282 & & .001 & 0.062 \\
Nsprints & .284 & & .001 & 0.061 \\
Steps/min & $.000^{* *}$ & A,B,C,D,F & .108 & $\mathbf{0 . 6 9 5}$ \\
Jumps/min & $.018^{*}$ & A & .007 & 0.170 \\
PLmáx & $.000^{* *}$ & A,B,C & .021 & 0.295 \\
PL/min & $.000^{*}$ & A,B,C,D & .088 & $\mathbf{0 . 6 2 1}$ \\
\hline
\end{tabular}

$A=1 v s 0-1 v s 1 ; B=1 v s 0-2 v s 2 ; C=1 v s 0-3 v s 3 ; D=1 v s 0-O f f+1 ; E=1 v s 1-2 v s 2 ; F=1 v s 1-3 v s 3$; $G=1 v s 1-O f f+1 ; H=2 v s 2-3 v s 3 ; l=2 v s 2-O f f+1 ; J=3 v s 3-O f f+1$. ${ }^{*} p<.05 ;{ }^{* *} p<.01$. as .50 in bold

A continuación, se presenta en la Tabla 5 las diferencias entre las situaciones de juego en todas las variables de CE analizadas en el programa DIB. Se han encontrado diferencias estadísticamente significativas entre las situaciones de juego en las variables $\mathrm{m} / \mathrm{min}$, acc/min, dece/min, $V_{\text {máx, }} V$, walk, jog, steps/min, jumps/min y $P L / m i n$. La tarea que más se diferencia de las demás es $1 \mathrm{vs} 0$. Los tamaños del efecto son pequeños en todas las variables excepto en $\mathrm{M} / \mathrm{min}$, Steps/min y PL/min. 
Tabla 5. Diferencias, tamaño del efecto y potencia entre las diferentes situaciones de juego en el programa de enseñanza DIB.

\begin{tabular}{lllrl}
\hline & $p$ & Post-Hoc & $\eta^{2}$ & \multicolumn{2}{c}{$d$} \\
\hline M/min & $.000^{* *}$ & B,C,D,E,F,G,I & .111 & 0.707 \\
Acc/min & $.046^{*}$ & & .005 & 0.145 \\
Dece/min & $.035^{*}$ & & .006 & 0.153 \\
V máx $^{*}$ & $.003^{* *}$ & B,E,H & .011 & 0.211 \\
V & $.000^{* *}$ & A,B,C,D,G,I & .040 & 0.410 \\
HIA & .380 & & .000 & 0.027 \\
Walk & $.000^{* *}$ & A,B,C,D & .030 & 0.351 \\
Jog & $.000^{* *}$ & A,B,C,D & .032 & 0.363 \\
Run & .385 & & .000 & 0.024 \\
Sprint & .083 & & .004 & 0.126 \\
Nsprints & .120 & & .003 & 0.111 \\
Steps/min & $.000^{* *}$ & A,B,C,D,E,F,G & .100 & 0.665 \\
Jumps/min & $.000^{* *}$ & B,C,D,E,G & .038 & 0.396 \\
PLmáx & .080 & & .004 & 0.126 \\
PL/min & $.000^{* *}$ & B,C,D,E,F,G & .082 & 0.597 \\
\hline
\end{tabular}

$A=1 v s 0-1 v s 1 ; B=1 v s 0-2 v s 2 ; C=1 v s 0-3 v s 3 ; D=1 v s 0-O f f+1 ; E=1 v s 1-2 v s 2 ; F=1 v s 1-3 v s 3$; $G=1 v s 1-O f f+1 ; H=2 v s 2-3 v s 3 ; l=2 v s 2-O f f+1 ; J=3 v s 3-O f f+1$.

${ }^{*} p<.05 ;{ }^{* *} p<.0$. d .50 en negrita

\section{DISCUSIÓN}

El objetivo del trabajo fue analizar las diferencias en las variables de la CE que soportan los alumnos durante las sesiones de EF según la situación de juego y el método de enseñanza en baloncesto en el contexto escolar. Los resultados obtenidos muestran que la metodología TGB obtiene valores más altos en variables que determinan la CE que la metodología DIB como en estudios anteriores (Bendiksen et al., 2014; Ingul, Tjonna, Stolen, Stoylen, \& Wisloff, 2010; Sperlich et al., 2010). La diferencia entre ambas metodologías es mayor en las situaciones de juegos más simples. La diferencia entre ambas metodologías se reduce cuando aumenta la complejidad de la situación de juego. La intensidad de las sesiones del TGB es mayor que las del DIB por lo que su uso en el contexto escolar es más beneficioso para la salud de los alumnos.

El uso de metodologías basadas en el juego modifica y establece diferentes situaciones de aprendizaje para mejorar la formación de los alumnos (Climent et al., 2015). Esta progresión en la formación de los alumnos también incluye los contenidos técnico-tácticos y la condición física. Los resultados obtenidos en este estudio muestran que las tareas diseñadas bajo una metodología TGA tienen una mayor CE en situaciones de juego simples $1 \mathrm{vs} 0,1 \mathrm{vs} 1$ y Off+1 que aquellas pertenecientes a la perspectiva DIB. Estos resultados son similares a estudios anteriores (Bendiksen et al., 2014; Cañadas et al., 2011; Faude et al., 2010). El diseño de la tarea y la organización de los alumnos son variables que tienen modifican la CE, siendo variables de gran relevancia para que existan diferencias significativas entre cada metodología en las situaciones de juego más simples. El diseño de las tareas de entrenamiento es un proceso muy importante para la planificación tanto de las demandas físicas como de los requerimientos 
pedagógicos. Se ha demostrado que los cambios en las agrupaciones de los jugadores y la presencia de oponentes condiciona la carga de la tarea (GómezCarmona et al., 2019). Las diferencian entre el programa TGB y el programa DIB disminuyen al aumentar la complejidad de las situaciones de juego. Otro motivo para que existan diferencias entre los programas son los diferentes medios de entrenamiento que se pueden utilizar en las tareas. Los medios de iniciación al entrenamiento pueden ser: i) Ejercicio de Aplicación Simple; ii) Ejercicio de Aplicación Complejo; iii) Juego Simple Inespecífico; iv) Juego Simple Específico; v) Juego Complejo Inespecífico; vi) Juego Complejo Específico; vii) Predeporte o Deporte Adaptado/Reducid; viii) Deporte; ix) Competición; x) Práctica Mental (Ibáñez et al., 2016). El medio de iniciación al entrenamiento más utilizado en el programa TGB es el juego simple específico y el juego complejo específico. Por otro lado, el medio de iniciación al entrenamiento que predomina en el programa TGB es ejercicio de aplicación simple.

En ambos programas de intervención se observan tendencias similares en los resultados a pesar de la diferencia existente entre ambos. En el TGB y en el DIB, las situaciones de juego más simples (1vs0, 1vs1, Off+1) presentan una mayor CE que las situaciones más complejas (2vs2 y 3vs3). Esto es debido a que al aumentar el número de jugadores disminuye la intensidad de la tarea y por tanto disminuye también la CE (Conte, Favero, Niederhausen, Capranica, \& Tessitore, 2016). Las situaciones más simples (1vs0 y 1vs1) permiten a los jugadores involucrarse más en el juego, donde tiene una menor duración de las repeticiones y favorecen que las defensas o los ataques sean más agresivas. Cuando los jugadores tienen una participación continua en el juego o en la tarea que se les presenta, los esfuerzos que realizan son mayores, aumentando la intensidad de las acciones (Conte et al., 2016). Además, en las situaciones de juego individuales las soluciones que los jugadores tienen que realizar no dependen de la ayuda de otros compañeros, sino que dependiendo exclusivamente de las capacidades físicas y técnicas de estos.

Por otro lado, las situaciones de juego más complejas (2vs2 y 3vs3) en donde hay más presencia de compañeros y adversarios presentan una complejidad táctica mayor, siendo juegos menos condicionales y, por lo tanto, la CE es menor (Bendiksen et al., 2014; Harrison, Gill, Kinugasa, \& Kilding, 2013). Las situaciones de juego reducidas aumentan la práctica y la mejora de los elementos técnicos del juego, mientras que la interacción con mayor número de compañeros y oponentes mejora la toma de decisión en juego (Conte et al, 2016). Se han estudiado las diferencias en el aprendizaje deportivo en función de la metodología de enseñanza, mostrando efectos de aprendizaje a favor de las metodologías centradas en el juego, tanto en toma de decisión y ejecución de habilidades, así como en las conductas tácticas relacionadas (Ibáñez, Feu, Cañadas, González-Espinosa, \& García-Rubio, 2016).

En la literatura hay diversos trabajos que concluyen que la modificación de las situaciones de juego en las tareas de enseñanza y entrenamiento deportivo determina los comportamientos que realizan los jugadores, modificando la carga de entrenamiento y las acciones técnicas y tácticas que se realizan (Dellal, LagoPenas, Wong, \& Chamari, 2011; Gabbett, Jenkins, \& Abernethy, 2010; Ngo et al., 2012). Esta forma de trabajo es un método es comúnmente utilizado en la 
enseñanza de los deportes como el baloncesto (Conte, Favero, Niederhausen, Capranica, \& Tessitore, 2015). La elección que el docente hace de la metodología de enseñanza para sus sesiones modifica y altera las variables relacionadas con el aprendizaje, la condición física y la experiencia de los deportistas. De hecho, las mismas situaciones de juego pueden orientarse hacia una metodología u otra, cambiando los aprendizajes que realizan los alumnos (Cañadas, Ibáñez, García, Parejo, \& Feu, 2013). Los programas formativos de los profesores de EF tienen que resaltar la importancia de las diferencias entre diferentes diseños de tareas, tanto a nivel condicional y físico, como a nivel de aprendizaje técnico-táctico. Como recoge el currículum de EP el docente tiene que formar de forma integral al alumno, siendo la condición física parte de esta formación.

En muchos casos, los alumnos solo realizan actividad física en el contexto escolar, concretamente en el área de EF (Guerra, Nobre, da Silveira, \& Taddei, 2013). En España, la EF es obligatoria desde el inicio de la educación primaria (6 años) hasta el final de la secundaria (16 años) con una frecuencia de dos clases semanales de 60 minutos de duración. Sin embargo, la OMS (2003) recomienda un mínimo de 60 minutos al día de esta actividad física para los jóvenes en edad escolar para llevar un estilo de vida saludable y prevenir enfermedades cardiovasculares en la edad adulta, entre otras (Andersen et al., 2006; Trudeau, Shephard, Arsenault, \& Laurencelle, 2003). Recientemente se ha demostrado la importancia de la intensidad de esa actividad física en la mejora de la condición física y la prevención de enfermedades (Bendiksen et al., 2014; Ingul, et al., 2010; Sperlich et al., 2010).

Con todo lo anterior, se evidencia la importancia de aumentar la intensidad de las clases de EF. Para ello, los resultados del estudio muestran que la metodología TGA y las situaciones de juego más simples favorecen a una mayor intensidad de la sesión, incrementen las demandas físicas de la CE y mejorando la condición física de los estudiantes. Además, el uso de sesiones de EF donde los contenidos sean deportes con balón es mejor elección que el uso de los contenidos tradicionales de EF desde un punto de vista de la condición física y la salud (Bendiksen et al., 2014). Además, la existencia de períodos de descansos cortos, típicos de medios de iniciación como el juego, permite la aparición de mejoras en otros aspectos físicos distintos del aeróbico, como son los neuromusculares (Bishop, Girard, \& Mendez-Villanueva, 2011), favoreciendo, por ejemplo, la capacidad de repetir esprines de los deportistas.

\section{CONCLUSIÓN}

Los programas diseñados para el estudio trabajan situaciones de juego más sencillas propias de la iniciación deportiva. Los resultados ponen de manifiesto que estas situaciones de juego sencillas tienen valores más altos en CE que las situaciones de juego más complejas. Los docentes deben utilizar programas de enseñanza deportiva basados en la metodología Tactical Games para la iniciación del deporte, como el TGB, para la enseñanza del baloncesto. Con el uso de este programa de enseñanza diseñado bajo la metodología TGA los docentes favorecerán a un mayor conocimiento teórico y práctico. Además, las tareas están adaptadas al nivel de experiencia de los alumnos, predominando 
las situaciones $1 \mathrm{vs} 0$ y $1 \mathrm{vs} 1$, y con una mayor CE. La intensidad del programa TGB es más beneficiosa para la salud que otros programas más directivos al aumentar la intensidad de la clase de EF

A día de hoy, el sistema educativo español sólo garantiza dos horas semanales de EF. Ante esta escasez de tiempo para el desarrollo de las recomendaciones básicas para la salud de los escolares, se recomienda a los profesores que empleen las metodologías que impliquen un mayor beneficio físico y de aprendizaje, como los basados en la metodología TCA. Igualmente, es recomendable emplear programas de intervención que hayan sido diseñados exprofeso en la metodología que los profesores consideren mejor y que estén previamente validados.

\section{REFERENCIAS BIBLIOGRÁFICAS}

Alarcón, F., Cárdenas, D., Miranda, M. T., Ureña, N., \& Piñar, M. I. (2011). Influence of teaching model on the mobility in basketball. Revista Internacional De Medicina Y Ciencias De La Actividad Fisica Y Del Deporte, 11(44), 749-766.

Andersen, L. B., Harro, M., Sardinha, L. B., Froberg, K., Ekelund, U., Brage, S., \& Anderssen, S. A. (2006). Physical activity and clustered cardiovascular risk in children: a cross-sectional study (The European Youth Heart Study). Lancet, 368(9532), 299-304. doi:10.1016/s0140-6736(06)69075-2

Barrett, S., Midgley, A., \& Lovell, R. (2014). PlayerLoad (TM): Reliability, Convergent Validity, and Influence of Unit Position During Treadmill Running. International Journal of Sports Physiology and Performance, 9(6), 945-952. doi:10.1123/ijspp.2013-0418

Beato, M., Bartolini, D., Ghia, G., \& Zamparo, P. (2016). Accuracy of a $10 \mathrm{~Hz}$ GPS Unit in Measuring Shuttle Velocity Performed at Different Speeds and Distances (5-20 M). Journal of Human Kinetics, 54(1), 15-22. doi:10.1515/hukin-2016-0031

Bendiksen, M., Williams, C. A., Hornstrup, T., Clausen, H., Kloppenborg, J., Shumikhin, D., . . . Krustrup, P. (2014). Heart rate response and fitness effects of various types of physical education for 8- to 9-year-old schoolchildren. European Journal of Sport Science, 14(8), 861-869. doi:10.1080/17461391.2014.884168

Bishop, D., Girard, O., \& Mendez-Villanueva, A. (2011). Repeated-sprint abilityPart II. Sports medicine, 41(9), 741-756.

Borresen, J., \& Lambert, M. I. (2008). Quantifying Training Load: A Comparison of Subjective and Objective Methods. International Journal of Sports Physiology and Performance, 3(1), 16-30.

Cañadas, M., Ibáñez, S. J., Feu, S., García, J., \& Parejo, I. (2011). Análisis de los medios de entrenamiento en un equipo minibasket y la influencia de un programa formativo para el entrenador. Un estudio de caso. Ágora para la Educación Física y el Deporte, 13(3), 363- 382.

Cañadas, M., Ibáñez, S. J., García, J., Parejo, I., \& Feu, S. (2013). Game situations in youth basketball practices. Revista Internacional de Medicina y Ciencias de la Actividad Física y del Deporte, 13(49), 41-54. 
Conte, D., Favero, T. G., Niederhausen, M., Capranica, L., \& Tessitore, A. (2015). Physiological and technical demands of no dribble game drill in young basketball players. The Journal of Strength \& Conditioning Research, 29(12), 3375-3379.

Conte, D., Favero, T. G., Niederhausen, M., Capranica, L., \& Tessitore, A. (2016). Effect of different number of players and training regimes on physiological and technical demands of ball-drills in basketball. Journal of Sports Sciences, 34(8), 780-786. doi:10.1080/02640414.2015.1069384

Chen, A., Martin, R., Sun, H., \& Ennis, C. D. (2007). Is in-class physical activity at risk in constructivist physical education? Research Quarterly for Exercise and Sport, 78(5), 500-509.

Delextrat, A., Badiella, A., Saavedra, V., Matthew, D., Schelling, X., \& TorresRonda, L. (2015). Match activity demands of elite Spanish female basketball players by playing position. International Journal of Performance Analysis in Sport, 15(2), 687-703.

Dellal, A., Lago-Penas, C., Wong, D. P., \& Chamari, K. (2011). Effect of the number of ball contacts within bouts of 4 vs. 4 small-sided soccer games. International Journal of Sports Physiology and Performance, 6(3), 322333.

Faude, O., Kerper, O., Multhaupt, M., Winter, C., Beziel, K., Junge, A., \& Meyer, T. (2010). Football to tackle overweight in children. Scandinavian Journal of Medicine \& Science in Sports, 20, 103-110. doi:10.1111/j.16000838.2009.01087.x

Feliz, T., y Ricoy, M.C. (2002). El diseño y desarrollo del currículum: las adaptaciones curriculares. En J. González (Ed.), Necesidades educativas especiales e intervención psicopedagógica (pp. 85-119). Alcalá de Henares: Universidad de Alcalá, Servicio de Publicaciones.

Fernández, E., Cecchini, J., \& Zagalaz, M. L. (2002). Didáctica de la educación física en la educación primaria. Madrid: Síntesis.

Feu,S., García-Rubio, J., Gamero, M. G., \& Ibáñez, S. J. (2019). Task planning for sports learning by physical education teachers in the pre-service phase. Plos One, 14(1), 1-18. doi.org/10.1371/journal.pone.0212833

Field, A. (2009). Discovering Statistics Using SPSS. London: SAGE Publications Ltd.

Foster, C., Florhaug, J. A., Franklin, J., Gottschall, L., Hrovatin, L. A., Parker, S., ... Dodge, C. (2001). A new approach to monitoring exercise training. Journal of Strength and Conditioning Research, 15(1), 109-115. doi:10.1519/00124278-200102000-00019

Gabbett, T. J., Jenkins, D. G., \& Abernethy, B. (2010). Physiological and skill demands of 'on-side'and 'off-side'games. The Journal of Strength \& Conditioning Research, 24(11), 2979-2983.

García, J., Parejo, I., \& Cañadas, M.. (2010). Valoración de la carga de entrenamiento: Una experiencia real con un equipo de baloncesto de liga EBA. Revista Internacional de Deportes Colectivos, (5), 4-17.

Gómez-Carmona, C.D., Gamonales, J.M., Feu, S., \& Ibáñez, S.J. (2019). Estudio de la carga interna y externa a través de diferentes instrumentos. Un estudio de casos en fútbol formativo. Sportis Sci J, 5 (3), 444-468.

González-Espinosa, S., Feu, S., García-Rubio, J., Antúnez, A., \& García-Santos, D. (2017). Diferencias en el aprendizaje según el método de enseñanza- 
aprendizaje en el baloncesto Revista de Psicología del Deporte, 26(1), 65-70.

González-Espinosa, S., Ibáñez, S. J., \& Feu, S. (2017). Diseño de dos programas de enseñanza del baloncesto basados en métodos de enseñanzaaprendizaje diferentes. E-balonmano.com: Revista de Ciencias del Deporte, 13(2), 131-152.

González-Espinosa, S., Ibáñez, S. J., Feu, S., \& Galatti, L. (2017). Intervention programs for sports education in the school context, PETB and PEAB: Preliminary study. Retos, 31, 107-113.

Guerra, P. H., Nobre, M. R., da Silveira, J. A., \& Taddei, J. A. (2013). The effect of school-based physical activity interventions on body mass index: a meta-analysis of randomized trials. Clinics, 68(9), 1263-1273. doi:10.6061/clinics/2013(09)14

Harrison, C. B., Gill, N. D., Kinugasa, T., \& Kilding, A. E. (2013). Quantification of physiological, movement, and technical outputs during a novel small-sided game in young team sport athletes. Journal of Strength and Conditioning Research, 27(10), 2861-2868. doi:10.1519/JSC.0b013e318280c98d

Harvey, S., Cushion, C. J., Wegis, H. M., \& Massa-Gonzalez, A. N. (2010). Teaching games for understanding in American high-school soccer: a quantitative data analysis using the game performance assessment instrument. Physical Education and Sport Pedagogy, 15(1), 29-54. doi:10.1080/17408980902729354

lbáñez, S. J. (2002). Los contenidos de enseñanza del baloncesto en las etapas de formación. En S. J. Ibáñez \& M. Macías (Eds.), Novos Horizontes para o treino do basquetebol. Lisboa: FMH.

Ibáñez, S. J., Feu, S., \& Cañadas, M. (2016). Integral analysis system of training tasks, SIATE, in invasion games. E-Balonmano com: Revista de Ciencias del Deporte, 12(1), 3-30.

Ibáñez, S. J., Feu, S., Cañadas, M., Gonzalez-Espinosa, S., \& García-Rubio, J. (2016). Estudio de los Indicadores de Rendimiento de Aprendizaje Tras la Implementación de un Programa de Intervención Tradicional y Alternativo Para la Enseñanza del Baloncesto. Kronos: revista universitaria de la actividad física y el deporte, 15(2), 1.

Ingul, C. B., Tjonna, A. E., Stolen, T. O., Stoylen, A., \& Wisloff, U. (2010). Impaired Cardiac Function Among Obese Adolescents. Archives of Pediatrics \& Adolescent Medicine, 164(9), 852-859.

McCormick, B. T., Hannon, J. C., Newton, M., Shultz, B., Miller, N., \& Young, W. (2012). Comparison of Physical Activity in Small-Sided Basketball Games Versus Full-Sided Games. International Journal of Sports Science \& Coaching, 7(4), 689-697. doi:10.1260/1747-9541.7.4.689

Mesquita, I., Farias, C., \& Hastie, P. (2012). The impact of a hybrid Sport Education-Invasion Games Competence Model soccer unit on students' decision making, skill execution and overall game performance. European Physical Education Review, 18(2), 205-219. doi:10.1177/1356336x12440027

Metzler, M. W. (2011). Instructional models for physical education. Scottsdale, Arizona: Holocomb Hathaway.

Miller, A. (2015). Games Centered Approaches in Teaching Children \& Adolescents: Systematic Review of Associated Student Outcomes. 
Journal of Teaching in Physical Education, 34(1), 36-58. doi:10.1123/jtpe.2013-0155

Mitchell, S. A., Oslin, J. L., \& Griffin, L. L. (1997). Teaching sport concepts and skills: A Tactical Game Approach. Leeds, United Kingdom: Human Kinetics.

Morgan, G., Muir, B., \& Abraham, A. (2014). Systematic observation. In L. Nelson, R. Groom, \& P. Potrac (Eds.), Research Methods in Sports Coaching (pp. 123-131). London: Routledge.

Muñoz-Lopez, A., Granero-Gil, P., Pino-Ortega, J., \& De Hoyo, M. (2017). The validity and reliability of a 5-hz GPS device for quantifying athletes' sprints and movement demands specific to team sports. Journal of Human Sport and Exercise, 12(1), 156-166. doi:10.14198/jhse.2017.121.13

Ngo, J. K., Tsui, M.-C., Smith, A. W., Carling, C., Chan, G.-S., \& Wong, D. P. (2012). The effects of man-marking on work intensity in small-sided soccer games. Journal of sports science \& medicine, 11(1), 109.

O'Hara, J. P., Brightmore, A., Till, K., Mitchell, I., Cummings, S., \& Cooke, C. B. (2013). Evaluation of Movement and Physiological Demands of Rugby League Referees Using Global Positioning Systems Tracking. International Journal of Sports Medicine, 34(9), 825-831. doi:10.1055/s0033-1333694

Organización Mundial de la Salud. (2010). Recomendaciones mundiales sobre la actividad física para la salud. Recuperado: http://whqlibdoc.who.int/publications/2010/9789243599977_spa.pdf.

Pill, S. (2015). Implementing game sense coaching approach in australian football through action research. Ágora para la educación física y el deporte, 18(1), 1-19.

Reina, M., Mancha, D., \& Ibáñez, S. J. (2017). ¿ Se entrena como se compite? Análisis de la carga en baloncesto femenino. Revista de Psicología del Deporte, 26(1), 9-13.

Reina, M.; Mancha-Triguero, D.; García-Santos, D.; García-Rubio, J., e lbáñez, Sergio J. (2019). Comparación de tres métodos de cuantificación de la carga de entrenamiento en baloncesto. RICYDE. Revista internacional de ciencias del deporte. 58(15), 368-382. https://doi.org/10.5232/ricyde2019.05805

Sperlich, B., Zinner, C., Heilemann, I., Kjendlie, P.-L., Holmberg, H.-C., \& Mester, J. (2010). High-intensity interval training improves VO2peak, maximal lactate accumulation, time trial and competition performance in 9-11-yearold swimmers. European Journal of Applied Physiology, 110(5), 10291036. doi:10.1007/s00421-010-1586-4

Thalheimer, W., \& Cook, S. (2002). How to calculate effect sizes from published research: A simplified methodology. Work-Learning Research.

Thomas, J. R., Silverman, S., \& Nelson, J. (2015). Research methods in physical activity, 7E: Human kinetics.

Torres-Ronda, L., Ric, A., Llabres-Torres, I., de Las Heras, B., \& Schelling, X. (2016). Position-dependent cardiovascular response and time-motion analysis during training drills and friendly matches in elite male basketball players. The Journal of Strength \& Conditioning Research, 30(1), 60-70.

Trudeau, F., Shephard, R. J., Arsenault, F., \& Laurencelle, L. (2003). Tracking of physical fitness from childhood to adulthood. Canadian Journal of Applied 
Rev.int.med.cienc.act.fís.deporte - vol. 20 - número 79 - ISSN: 1577-0354

Physiology-Revue Canadienne De Physiologie Appliquee, 28(2), 257-271. doi:10.1139/h03-020

Número de citas totales / Total references: 51 (100\%)

Número de citas propias de la revista / Journal's own references: 2 (3,92\%)

Rev.int.med.cienc.act.fís.deporte - vol. X - número X - ISSN: 1577-0354 